\title{
Feline Lymphoplasmacytic Gingivostomatitis: Clinical and Anatomopathological Aspects
}

\author{
Eduardo Rebelato Sakis', Tanise Policarpo Machado', Diorges Henrique Setim ${ }^{1,2}$, \\ Luciane Melatti ${ }^{3}$ \& Adriana Costa da Motta ${ }^{1,2}$
}

\begin{abstract}
Background: Feline lymphoplasmacytic gingivostomatitis (FLPG) is an idiopathic, multifactorial and highly challenging condition, since the results of the available treatments are partial and transitory. A set of lesions triggered by inflammatory reaction is observed, sometimes with ulcerations in the oral mucosa, leading to algia and discomfort. Histopathological examination figures as the definitive diagnostic tool, presenting distinct cellular elements. The present study aims to report three cases of FLPG, firstly addressing the anatomopathological findings, as well as the therapeutic approach used and the results obtained in each case.

Cases: Case 1- Occurred in a feline Siamese male 15-year-old, weighing $3.8 \mathrm{~kg}$, which was admitted at a local clinic, under the complaint of prostration, dysphagia, and weight loss, signs that were noticed approximately 5 days before. Clinical evaluation revealed multiple proliferative and ulcerative lesions, extending until the isthmus of the fauces, erythematous areas around the teeth and multifocal gingival reabsorption. Once the clinical signs and lesions were observed, samples were collected for histopathological examination and sent to the Laboratory of Animal Pathology (LAP) of Faculty of Agronomy and Veterinary Medicine of the University of Passo Fundo (FAVM-UPF). Histologically, both fragments presented lymphoplasmacytic infiltration, with large and intensely eosinophilic cytoplasm, often with granular aspect, central to lateralized hyperchromatic nuclei, located predominantly in lamina propria and mucosa, as well as a discrete Mott cell infiltrate. It was also observed the presence of diffuse suppurative chronic inflammatory infiltrate, predominantly in lamina propria and mucosa, as well as foci of edema and hemorrhage, in addition to loss of tissue architecture establishing the definitive diagnosis of FLPG. The tutor went to another veterinarian for a second opinion, but the patient died within four months. Case 2- Occurred in a no defined-breed male feline 7-year-old, weighing $3.9 \mathrm{~kg}$, on complaint of hyporexia, inappetence, progressive thinning and greasy-looking fur. The tutor reported that the patient showed an increasing disinterest for offered feed, for approximately three weeks, but in recent days, consumption had declined dramatically. Clinical evaluation showed the same pattern of lesions in case 1, but with higher intensity, especially in the fauces area, as well as multifocal periodontal lesion and gingival retraction areas in several teeth. After the clinical signs and lesions were observed, samples were collected for histopathological examination and sent to the LAP of the FAVM-UPF, having the same diagnosis of case 1. The patient was submitted to a complete teeth extraction and, up to date, is asymptomatic, with a positive evolution. Case 3- Occurred in a no defined-breed female feline 6-year-old, weighing $4.1 \mathrm{~kg}$, showing bleeding gums, reluctance to eat and mild halitosis, signs noticed around two weeks before. The patient was submitted to clinical evaluation, with lesions that consisted of erythematous areas, more evident in molar teeth, as well as ulcerated foci of the oral mucosa. Samples were collected for histopathological examination and sent to the LAP of the FAVM-UPF, with the same diagnosis as previous cases. The therapeutic approach used consisted of immunodulation and immunosuppression, using cyclosporin and prednisolone, respectively. Up to present, the patient shows control on severity of lesions, maintaining a good life quality. Discussion: The present study observed that clinical and anatomopathological findings corroborate with those cited in the literature, with special emphasis on the presence of Mott cells as well as the marked lymphoplasmacytic infiltrate, common in all three cases. The results of chosen treatments showed variation in results, even with the individualization of the therapeutic approach in the three cases.
\end{abstract}

Keywords: oral cavity, ulceration, chronic infiltrate, Mott cells, periodontitis.

Descritores: cavidade oral, ulceração, infiltrado crônico, células de Mott, periodontite. 


\section{INTRODUCTION}

Gingiostomatitis are common pathologies for feline clinic routine $[1,2,5,9,10]$, referred as the second most frequent oral disease in the species, shortly after periodontal disease, with special relevance for FLPG $[1,9]$. It is an idiopathic disease and is believed to be multifactorial $[8,10-12,14]$. The disease presents aggressive behavior [5], characterized by intense inflammation, erythema, ulceration areas and gingival hyperplasia $[1,2,5,7,11]$, eventually reaching glossopalatine arches (isthmus of the fauces), palate, and bucal mucosa $[2,5,7,11,12,14]$. Odontoclastic reabsorption is frequently observed due to the inflammatory reaction, which may lead to dental loss [1,2,11].

The presumptive diagnosis can be achieved through physical examination, evaluation of clinical history, disease evolution and previous treatments [11]. Due to the algia related to the clinical condition, anesthesia or sedation may be necessary to accomplish an appropriate physical examination [11]. The definitive diagnosis is established by histopathology, which reveals the presence of lymphoplasmacytic infiltrate $[1,6,7,14]$, often accompanied by Mott cells and mast cell infiltrate.

Regardless of being a frequent pathology in feline clinics, laboratorial diagnosis is not always executed as suggested. Thereby, the present report aims to document three cases of FLPG, addressing its clinical and pathological findings, emphasizing the differential diagnosis with other feline oral cavity diseases, as well as the therapeutic approach applied.

\section{CASES}

Case 1- Occurred in a feline Siamese male 15-year-old, weighing $3.8 \mathrm{~kg}$, which was admitted at a local clinic, under the complaint of prostration, dysphagia, and weight loss, signs that were noticed approximately 5 days before. Clinical evaluation revealed multiple proliferative and ulcerative lesions, extending until the isthmus of the fauces, erythematous areas around the teeth and multifocal gingival reabsorption. Once the clinical signs and lesions were observed, samples were collected for histopathological examination and sent to the Laboratory of Animal Pathology (LAP) of Faculty of Agronomy and Veterinary Medicine of the University of Passo Fundo (FAVM-UPF), The samples consisted of fragments of lesions on the base of tongue measuring 0.5 to $1 \mathrm{~cm}$ in diameter, of indefinite shape, irregular surface, firm consistency and brownish color. Histologically, both fragments presented lymphoplasmacytic infiltration, with large and intensely eosinophilic cytoplasm, often with granular aspect, central to lateralized hyperchromatic nuclei, located predominantly in lamina propria and mucosa, as well as a discrete Mott cell infiltrate (Figure 1A). Pleomorphism was moderate and the mitotic index was low. There were few areas of intact epithelium for proper evaluation, as there were extensive areas of ulceration (Figure 1B). It was also observed the presence of diffuse suppurative chronic inflammatory infiltrate, predominantly in lamina propria and mucosa, as well as foci of edema and hemorrhage, in addition to loss of tissue architecture. The histochemical technique of Toluidine Blue evidenced cytoplasmatic granule staining in some cells, sparse and diffuse throughout the tissue (Figure 1C). The tutor went to another veterinarian for a second opinion, but the patient died within four months.

Case 2- Occurred in a no defined-breed male feline 7-year-old, weighing $3.9 \mathrm{~kg}$, on complaint of hyporexia, inappetence, progressive thinning and greasylooking fur. The tutor reported that the patient showed
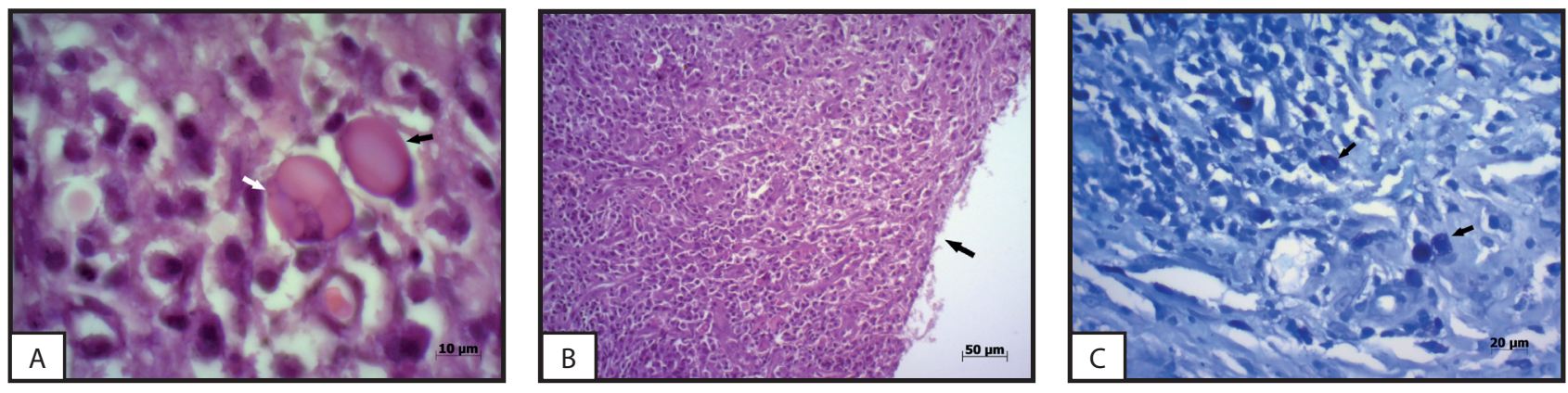

Figure 1. Feline lymphoplasmacytic gingivostomatitis - Case 1. A- Oral submucous layer. Mott cells containing multiple Russel bodies (white arrow) and single (black arrow) [HE; 1000x]. B- Mucous and oral submucosal layers. Presence of a marked lymphoplasmacytic infiltrate; on the right side, it is observed a focally extensive ulceration, with loss of mucosal layer and exposure of the submucosa (arrow) [HE; 200x]. C- Submucosal layer. Cells with intracytoplasmic granules stained in purple - mastocyte infiltrate (arrows) [Toluidine Blue; 400x]. 
an increasing disinterest for offered feed, for approximately three weeks, but in recent days, consumption had declined dramatically. Clinical evaluation showed the same pattern of lesions in case 1, but with higher intensity, especially in the fauces area, as well as multifocal periodontal lesion and gingival retraction areas in several teeth. After the clinical signs and lesions were observed, samples were collected for histopathological examination and sent to the LAP of the FAVM-UPF. An oral mucosa fragment was received, measuring $1 \mathrm{~cm}$ on its largest axis, of indefinite shape, irregular surface, firm consistency, solid appearance and whitish color. At microscopy, severe diffuse lymphoplasmacytic infiltrate was observed, extending throughout the epithelial/ lamina propria and submucosal interface region, as well as a moderate Mott cell infiltrate (Figure 2). Discrete multifocal chronic suppurative infiltrate, areas of necrosis and epithelial ulceration, hyperkeratosis and multifocal acanthosis were also observed. The patient was submitted to a complete teeth extraction and, up to date, is asymptomatic, with a positive evolution.

Case 3-Occurred in a no defined-breed female feline 6-year-old, weighing $4.1 \mathrm{~kg}$, showing bleeding gums, reluctance to eat and mild halitosis, signs noticed around two weeks before. The patient was submitted to clinical evaluation, with lesions that consisted of erythematous areas, more evident in molar teeth, as well as ulcerated foci of the oral mucosa. Samples were collected for histopathological examination and sent to the LPA of the FAMV-UPF, measuring approximately $1.0 \mathrm{~cm}$ on its larger axis, of indefinite shape, irregular surface, firm consistency, solid appearance and whitish

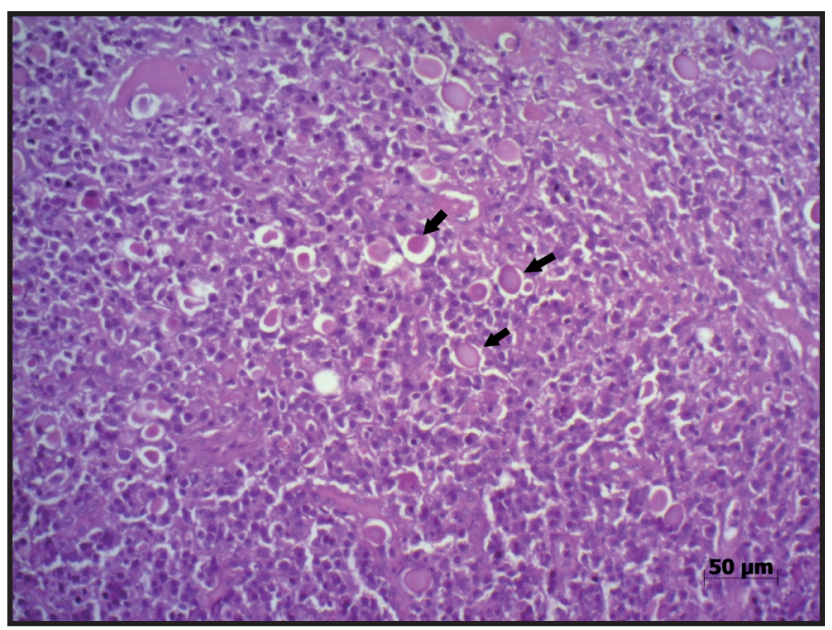

Figure 2. Feline lymphoplasmacytic gingivostomatitis - Case 2. Oral submucosal layer. Presence of marked lymphoplasmocytic infiltrate, in addition to plasmocytes with abundant eosinophilic cytoplasm (Mott cells - arrows) [HE; 200x]. color. At microscopy, diffuse lymphoplasmacytic infiltrate was observed in both fragments, extending throughout the epithelial / lamina propria region and deep submucosa, as well as muscle region, and sparse Mott cells (Figure 3). There was, also, moderate chronic multifocal suppurative infiltrate, areas of epithelial ulceration, hyperkeratosis and multifocal acanthosis. The therapeutic approach used consisted of immunodulation and immunosuppression, using cyclosporin and prednisolone, respectively. Up to present, the patient shows control on severity of lesions, maintaining a good life quality.

\section{DISCUSSION}

The onset of FLPG is related to a deficient reaction of the immune system to oral antigens or to an exacerbated immune response to these $[1,4,14]$, increase on bacterial plaque in dentition [9] and diet [2]. Bacteria and their metabolites trigger an inflammatory reaction [8], with lymphocytes and plasma cells being the first cells to arise and predominate in damaged tissue [2]. Some viral infections have been suggested as primary or aggravating factors of FLPG, being objects of frequent studies, such as calicivirus (FCV), feline immunodeficiency virus (FIV), feline leukemia virus (FeLV) and feline herpesvirus (FHV), however results are quite variable and, therefore, inconclusive. In addition, bacteria of the genus Bartonella spp. may also be involved $[7,11,14]$.

In order to aid in the definitive diagnosis, it is possible to request complementary tests, such as: proteinogram, since the disease can present

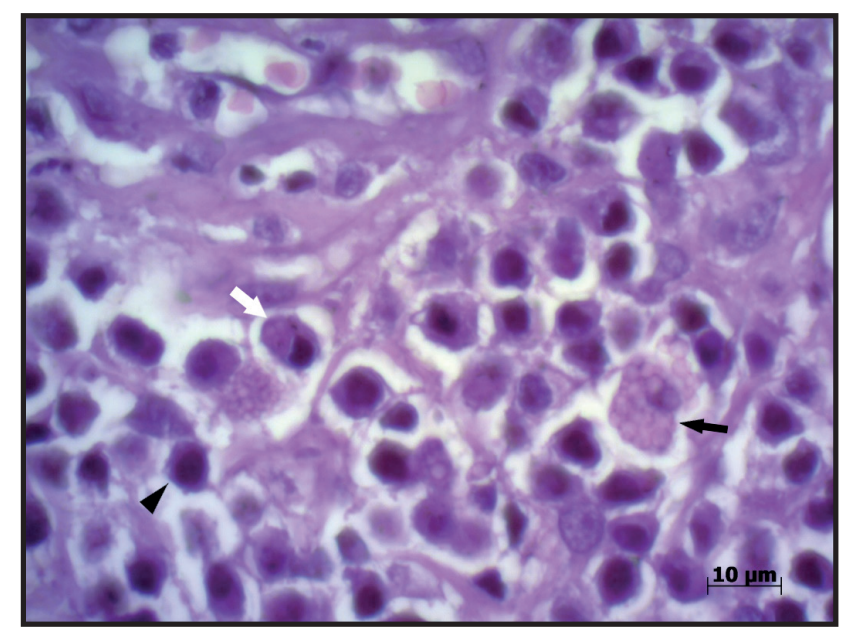

Figure 3. Feline lymphoplasmacytic gingivostomatitis - Case 3. Oral submucous layer. Presence of plasma cell infiltrate (white arrow), lymphocytic infiltrate (arrow head), as well as Mott cell containing multiple Russel bodies (black arrow) [HE; 1000x]. 
hyperproteinemia due to hypergammaglobulinaemia [7,9], consistent with chronic antigenic stimulation [6], serological and virological analyzes, radiographic examination to identify odontoclastic reabsorption lesions, periodontal disease and eventual dental tips retained within the alveoli [7,9].

The development of FLPG leads to characteristic macroscopic and microscopic findings, presenting distinct lesions and cellular components. The affected animals exhibit varying degrees of inflammatory lesion, usually showing worsening with the evolution of the disease [7] and causing ulceration and/or proliferation of the local epithelium. Clinical signs consist of halitosis, anorexia, inappetence, dysphagia, selective appetite, variable degree of algia, weight loss, dehydration, ptyalism, with eventual associated hemorrhage, chewing of face, reduction of toilet habits and, sometimes, exteriorization of tongue [1,2,5-7,9-11]. Macroscopic lesions tend to be multifocal to diffuse, not limited to a single mass [5], being bilateral and symmetrical, eventually presenting hemorrhagic areas. The macroscopic lesions, as well as clinical signs, were identical to those found on the three patients, consisting of proliferative foci of the gingival epithelium, erythematous and ulcerative lesions distributed in the oral cavity, and gingival retraction, more pronounced in case 2 . The inflammatory process can cause gingival retraction [11], which was presented in moderate intensity in case 2, therefore leading to the option for surgical treatment, with complete teeth extraction.

FLPG shows no seeming sexual predisposition, with similar occurrence between males and females. The Siamese race is commonly mentioned as one of the most affected, but Persian, Abyssinian, Himalayan and Burmese races are also frequently compromised. The average age of animals is of 8 to 8.8 years, varying between 3 and 15 years $[2,9,10,11]$. The epidemiological data corroborates with the present report, especially when referring to gender and age.

The most commonly observed histological pattern consists of marked lymphoplasmacytic inflammation, mostly on the transition region between the mucosa and the submucosa [10,11], as well as the presence of lymphocytes, mast cells, macrophages and neutrophils, these last ones in ulcerated areas. All of the aforementioned elements were observed in all three patients, confirming other reports [2,5,10-12,14]. It was also observed intense hyperplasia, with deep areas of ulceration [2], as well as parakeratosis and vacuolar degeneration [10], lesions noted on the three reported cases. Areas with formation of granulation tissue [11] and, eventually, binucleate cells [12] and eosinophils [11] may also be observed. Multifocal areas of granulation tissue formation were noted and, only in case 2, a small and sparse population of binucleate cells was found.

The presence of scattered Mott cells diffuse on the evaluated material was verified in all three patients, corroborating with other studies that mention these as frequently present [10-12]. Mott cells are plasma cells containing Russel bodies in their cytoplasm. These entities represent spherical inclusions of immunoglobulins, which may appear individually or multiple in the cytoplasm [3], displacing the nucleus to the periphery of the cell. Mott cells and Russel bodies originate in reactive plasmacytosis [3]. Thus, the presence of such cells corroborates with the chronic and immunomediated character of FLPG [10-12].

The usage of histochemical technique of toluidine blue allows the disclosure of mast cell infiltrate, which is often discrete to moderate, distributed mostly in the submucosa and oral epithelium [10]. The mast cell infiltrate was found in all three cases with the reffered intensity. It is suggested that the presence of mast cells may be related with the pathogenesis of oral inflammation in felines [10].

The histopathological examination is of extreme value and was fundamental for differential diagnosis with other pathologies, such as uremic gingivitis [2], diabetes mellitus [9], neoplasms, periodontal disease, eosinophilic granuloma and autoimmune diseases [1].

FLPG does not have a well-known and fully effective treatment $[1,2,6,9,11,14]$. It is a pathology of chronic nature, presenting eventual exacerbations $[1,9]$. Therefore, therapeutic approaches have as main objective to decrease inflammation and, consequently, improve the patient's life quality $[1,11]$. To do so, it is possible to choose for clinical or surgical approaches, or a combination of both $[1,2,11,14]$, although results are variable and duration, unpredictable. Thus, it is recommended to adapt available therapies depending on each case $[1,9,11,14]$.

In order to achieve this objective, it was suggested a proper oral hygiene in cases 2 and 3, aiming at the reduction of bacterial plaque and, consequently, the decline of the inflammatory stimulus, a conduct proposed by some studies $[1,2,6,7,9,11]$. It has been 
recommended the introduction of diets that reduce tartar formation and present hypoallergenic characteristics [1,9].

In cases of patients showing gingival retraction, periodontal disease and/or mobility, the removal of all premolar and molar teeth is suggested. If there is not a full cease of symptoms, the extraction should be complete $[1,2,7,14]$, seeking to remove even fragments of dental roots [2]. Such conduct was used in the patient of case 2 , which presented an adequate response, with removal of symptoms.

Since there is not a guaranteed long-term cure, other therapeutic approaches are suggested, according to their action mechanism, such as thalidomide (anti-inflammatory, with effects on viral replication, and acceleration of healing process) [14]; cyclosporin [9,11], feline recombinant Omega interferon and recombinant human interferon alpha-24 (immunomodulators) $[9,11,14]$; lactoferrin (bacterial growth inhibitor) [9,14]; prednisolone (immunosuppressant) [9,11,14]; piroxicam (anti-inflammatory) [11,14]; carbon dioxide laser (removal of proliferating tissue and stimulative of healing process) [14]; and gold salts (anti-inflammatory effects, used eventually in cases of autoimmune disease) [11].

The prognosis of this syndrome is commonly considered reserved, since the results are not guaranteed by the therapies available, as well as by the constant recurrences that patients may present $[1,6]$. The multifactorial character of FLPG is also an aggravating factor, since it requires that veterinarians and tutors commit themselves to a care routine, aiming at the improvement of patients' life quality, controlling or reducing lesions and clinical signs of the disease.

It is concluded, therefore, that clinical and anatomopathological findings corroborate with those cited by literature, with special emphasis on the presence of Mott cells, as well as the marked lymphoplasmacytic infiltrate, common in all three cases. Similarly, the treatment, adapted for each case, presented variation in the results, even with the individualization of the therapeutic approach, with the exception of case 1 , which did not return to the responsible veterinarian for reevaluation.

Declaration of interest. The authors report no conflicts of interest. The authors alone are responsible for the content and writing of the paper.

\section{REFERENCES}

1 Allemand V.C., Radighieri R. \& Bearl C.A. 2013. Gengivite-estomatite linfoplasmocitária felina: relato de caso. Revista de Educação Continuada em Medicina Veterinária e Zootecnia do CRMV-SP. 11(3): 24-29.

2 Appollo F.H., Carvalho V.G.G. \& Gioso M.A. 2010. Complexo gengivite-estomatite-faringite dos felinos. Clínica Veterinária. 15(84): 44-52.

3 Bain B.J. 2009. Russell bodies and Mott cells. American Journal of Hematology. 84(8): 516-516.

4 Gelberg H.B. 2017. Alimentary system and the peritoneum, omentum, mesentery, and peritoneal cavity. In: Zachary J.F. \& McGavin M.D. (Eds). Pathology Basis of Veterinary Disease. 6th edn. Saint Louis: Elsevier, pp.324-411.

5 Munday J.S., Löhr C.V. \& Kiupel M. 2017. Tumors of the alimentary tract. In: Meuten D.J. (Ed). Tumors in Domestic Animals. 5th edn. Ames: Wiley Blackwell, pp.499-601.

6 Willard M.D. 2010. Distúrbios do sistema digestório. In Nelson R.W. \& Couto C.G. (Eds). Medicina Interna de Pequenos Animais. 4.ed. Rio de Janeiro: Elsevier, pp.351-484.

7 Niemiec B.A. 2010. Small Animal dental, oral \& maxillofacial disease: a colored handbook. London: Manson Publishing Ltd., pp.60-69.

8 Niemiec B.A. 2013. The progression of disease. In: Niemiec B.A. (Ed). Veterinary periodontology. Ames: Wiley Blackwell, pp.41-68.

9 Niza M.M.R.E., Mestrinho L.A. \& Vilela C.L. 2004. Gengivo-estomatite crónica felina - um desafio clínico. Revista Portuguesa de Ciências Veterinárias. 99(551): 127-135.

10 Rolim V.M., Pavarini S.P., Campos F.S., Pignone V., Faraco C., Muccillo M.S., Roehe P.M., Costa F.V.A. \& Driemeier D. 2016. Clinical, pathological, immunohistochemical and molecular characterization of feline chronic gingivostomatitis. Journal of Feline Medicine and Surgery. 19(4): 1-7.

11 Roveredo C.D. 2018. Tratamento do complexo-gengivite-estomatite-faringite felino com implantes de ouro: estudo clínico piloto. 67f. Lisboa, Portugal. Dissertação (Mestrado em Medicina Veterinária), Universidade Lusófona de Humanidades e Tecnologias. 
12 Uzal F.A., Plattner B.L. \& Hostetter J.M. 2016. Alimentary System. In: Jubb K.V.F.V., Kennedy P.C. \& Palmer N.C. (Eds). Pathology of Domestic Animal. 6th edn. Saint Louis: Elsevier, pp.1-257.

13 Verstraete F.J.M. \& Lommer M.J. 2012. Principles of exadontics. In: Verstraete F.J.M. \& Lommer M.J. (Eds). Oral and maxillofacial surgery in dogs and cats. London: Saunders-Elsevier, pp.97-114.

14 Winer J.N., Arzi B. \& Verstraete F.J.M. 2016. Therapeutic management of feline chronic gingivostomatitis: a systematic review of the literature. Frontiers in Veterinary Science. 3(54): 1-10. 